\section{EL TURISMO DE NATURALEZA: UN PRODUCTO TURÍSTICO SOSTENIBLE}

\author{
Violante Martínez Quintana \\ Universidad Nacional de Educación a Distancia \\ ORCID iD: http://orcid.org/0000-0001-6208-1318 \\ vmartin@poli.uned.es
}

\section{NATURE TOURISM: A SUSTAINABLE TOURISM PRODUCT}

Cómo citar este artículo/Citation: Martínez Quintana, V. (2017). El turismo de naturaleza: un producto turístico sostenible. Arbor, 193 (785): a396. doi: http://dx.doi.org/10.3989/arbor.2017.785n3002
Copyright: (c) 2017 CSIC. Este es un artículo de acceso abierto distribuido bajo los términos de la licencia Creative Commons Attribution (CC BY) España 3.0.
RESUMEN: El turismo de naturaleza se ha conformado según los planteamientos académicos y las políticas turísticas en orden a un proceso lógico de evolución experimentada en el sector turístico en todo el mundo. Los impactos negativos que ha ocasionado el modelo convencional han puesto la voz de alarma, y todo el sector ha sido coadyuvado por el proceso general de la sostenibilidad en el desarrollo local, imperando en consecuencia el paradigma de la responsabilidad y sostenibilidad en los destinos turísticos. Tras un repaso conceptual del turismo de naturaleza convertido en un turismo alternativo, el presente artículo aporta las tendencias producidas en general, y la percepción de los paisajes culturales como producto turístico en particular. Finalmente se efectúa un análisis de las iniciativas para el fomento de un turismo de naturaleza alternativo y se ofrece una propuesta de planificación basada en el modelo de turismo responsable y sostenible, que actúe con garantía en el patrimonio natural y cultural como producto turístico sostenible.

PALABRAS CLAVE: Turismo de naturaleza; ecoturismo; desarrollo sostenible; planificación; producto turístico.
ABSTRACT: Nature tourism has emerged in the tourism field as a result of a logical evolution in line with public policies and academic research. After negative outcomes from traditional models first raised the alarm, the entire sector has tried to foster local development based on models of responsibility and sustainability. This article revises key concepts of nature based tourism and shows new tendencies and the perception of cultural landscapes that are seen as tourism products. Finally, it concludes by analysing new tendencies to foster alternative nature - based tourism. It also presents a planning proposal based on a responsible and sustainable tourism model to guarantee a sustainable tourism product within the natural and cultural heritage context.

KEYWORDS: Nature tourism; ecotourism; sustainable development; planning; tourism products. 


\section{INTRODUCCIÓN}

El turismo de naturaleza supone una enorme oportunidad de contactar con las raíces naturales y saludables que las personas necesitan en los hábitats en los que conviven, y puede actuar de compensación ante la "comodidad" del hábitat urbano polucionado y su ritmo de vida estresante. El desarrollo y progreso de la vida moderna han desgastado el medio ambiente y actuado con impactos negativos a través del modelo de turismo convencional. El Informe Brundtland definía a finales de los ochenta la sostenibilidad como "el desarrollo que satisface las necesidades de las generaciones presentes sin comprometer la capacidad de las generaciones futuras para satisfacer sus propias necesidades" (Comisión Mundial del Medio Ambiente y del Desarrollo y Brundtland, 1989, p. 23).

Pese al esfuerzo que se está llevando a cabo se ha hecho patente la pérdida de diversidad biológica, la destrucción de los hábitats, su sobre-explotación y la descarga de substancias dañinas que ocasionan pérdidas irreversibles de recursos. El ejemplo de Belice en el Estado del mismo nombre es muy ilustrativo ya que es conocida como una ciudad con ecoturismo mundial, que en sus inicios empezó como una industria con principios protectores del medioambiente y de las personas, y se convirtió en una zona turística con una industria muy competitiva. En 2012 tenía 341.000 habitantes y en 2016 ya había recibido más de un millón de visitantes. El turismo es la segunda prioridad del gobierno de Belice después de la agricultura, con un $44 \%$ de sus recursos terrestres y marítimos bajo protección y 92 reservas de la naturaleza.

Estos hechos forman parte de un todo que se ha debatido en la bibliografía especializada, que demanda urgentemente el asentamiento definitivo de la sostenibilidad económica, social y medioambiental para todo el sector turístico. Las Naciones Unidas han proclamado 2017 Año Internacional del Turismo Sostenible para el Desarrollo, un turismo bien concebido y gestionado, que forme parte de la Nueva Agenda 2030. Los tres Objetivos de Desarrollo Sostenible (ODS) que más implicación tienen son el Objetivo 8 que atiende a promover el crecimiento económico, inclusivo y sostenible, el empleo pleno y productivo y el trabajo decente para todos, el Objetivo 12 que representa el consumo y la producción responsables y el Objetivo 14, que se dedica a conservar y utilizar sosteniblemente los océanos, los mares y los recursos marinos para el desarrollo sostenible.

Las cifras de turismo en 2016 muestran un aumento en las llegadas de turistas internacionales de un 5\% entre enero y abril (Organización Mundial del Turismo (UNWTO). Panorama OMT del turismo internacional), según el barómetro OMT del Turismo Mundial del mes de junio. Con estas tendencias de crecimiento favorables la Organización Mundial del Turismo (OMT) ha seguido reforzando los objetivos para contribuir y mejorar el crecimiento económico, el desarrollo inclusivo y la sostenibilidad medioambiental en todas las agendas nacionales e internacionales. Las dimensiones medioambientales se incluyen en el marco estadístico de alcance internacional para medir el papel del turismo en el desarrollo sostenible, en las que se incluyen los indicadores de los Objetivos de DesarroIlo Sostenible (ODS) y los de la Red Internacional de Observatorios de Turismo Sostenible de la OMT. Un dato revelador es la iniciativa pionera en Madrid (España) sobre el sector turístico ante los Objetivos de Desarrollo Sostenible: turismo responsable, un compromiso para todos.

El trabajo que aquí se presenta tiene como objetivo general ofrecer una panorámica amplia de los planteamientos académicos y de las políticas turísticas que sobre el turismo de naturaleza se han llevado a cabo en el proceso de evolución del sector turístico en el mundo. Abarca un marco conceptual y los retos que ofrece para conocer la dimensión alcanzada, y sus principales características y consecuencias en los destinos turísticos. Es una reflexión de las definiciones y concepciones que se han ido elaborando, y que han pasado del modelo convencional del turismo al modelo alternativo más amplio y dinámico.

Desde una perspectiva más concreta, el lector podrá conocer la percepción que los turistas tienen de los paisajes culturales como producto turístico, sus fortalezas, debilidades, oportunidades y amenazas, que como aportación empírica intenta aproximarse a la realidad que presenta a través de sus opiniones y experiencias. Finalmente se efectúa un análisis de las iniciativas que se han fomentado en el turismo de naturaleza, y se ofrece una propuesta de planificación asentada en el modelo de turismo responsable y sostenible.

\section{MARCO CONCEPTUAL DEL TURISMO DE NATURALEZA}

El turismo de naturaleza está relacionado directamente con el desarrollo y el turismo sostenible, y prácticamente está referido a los hábitats naturales y a su biodiversidad, donde adquieren gran relevancia los parques naturales, las reservas protegidas, el ecoturismo, el medio rural, el agroturismo y el involucramiento de la población perteneciente al área determinada de implantación turística. 
La importancia de este turismo se empieza a notar en los riesgos que la industria del turismo ha conllevado, y deja de ser considerado como una industria blanca, debido a los impactos sociales, culturales y ecológicos. Las críticas se decantaron en la década de los setenta del siglo XX, y hasta la década siguiente no se empieza a transformar la conceptualización del turismo como portador de graves riesgos para la sociedad.

Con el turismo de naturaleza surge el ecoturismo, que contribuye a la conservación del entorno y su población y, si bien no se da una definición compartida, al menos se está de acuerdo en cuatro ideas básicas: 1) viajar a entornos naturales vírgenes, 2) interiorizar la experiencia obtenida en entornos naturales puros y en contacto con gentes locales, 3) impulsar la conservación de los recursos naturales y 4) educar en valores medioambientales y culturales de los lugares visitados (Epler Wood, 2002). El ecoturista es un viajero que disfruta su tiempo de ocio con un sentido de la ética que le motiva para participar en trabajos de rehabilitación en lugares degradados, y ayuda en tareas para el desarrollo de las poblaciones locales.

La definición de ecoturismo de la Wikipedia incorpora el término de turismo ecológico vinculado a las actividades turísticas sostenibles, de preservación y apreciación del medio natural-cultural que van a percibir los viajeros. Promueve un turismo ético, de bienestar en las poblaciones locales, con una estructura específica y un funcionamiento empresarial, grupal y cooperativo con estos principios. A finales de la década de los ochenta, y a principios de los noventa la Sociedad Internacional de Ecoturismo (The International Ecoturism Society (TIES), Ecoturismo) marca un momento fundamental en la atención del turismo respecto al medio ambiente, la atención de catástrofes naturales, y la posterior preocupación del cambio climático. A partir de ahí la escalada de ascenso del ecoturismo es imparable y culmina con la proclamación del Año Internacional de Ecoturismo por la ONU en 2002, donde tuvo lugar la Declaración de Quebec (Canadá) sobre el ecoturismo, bajo el auspicio del Programa de las $\mathrm{Na}$ ciones Unidas para el Medio Ambiente (PNUMA) y la Organización Mundial del Turismo (OMT). Los principios que allí se plantearon se situaron en este orden:

- Contribución activa en la conservación del patrimonio natural y cultural.

- Inclusión de las comunidades locales e indígenas en su planificación, desarrollo, explotación y logro de su bienestar.
- Interpretación del patrimonio natural-cultural del destino para visitantes.

- Destinado a viajeros independientes y grupos de tamaño reducido prioritariamente.

La OMT define el turismo sostenible como aquel "que tiene en cuenta las repercusiones actuales y futuras, económicas, sociales y medioambientales para satisfacer las necesidades de los visitantes de la industria, del entorno y de las comunidades anfitrionas" (Organización Mundial del Turismo. Sustainable Development of Tourism. Definición). Todas las modalidades de turismo, especialmente el turismo de naturaleza, deben albergar los principios de sostenibilidad para el desarrollo y gestión de las zonas de destino turístico, la autenticidad sociocultural de las comunidades anfitrionas y la participación de todos los agentes.

El Instituto de Worldwatch (Chafe, 2005) distingue ocho categorías de turismo semejantes que tienen como referente la naturaleza y el medio ambiente, tales como turismo de aventura, ecoturismo, geoturismo, turismo masivo (mar, arena, sol), turismo basado en la naturaleza, turismo pro-pobres, turismo responsable y turismo sostenible.

El término ecoturismo y todas sus implicaciones conciernen básicamente a la conservación de la naturaleza y de los recursos naturales, y es un vocablo que se utiliza a nivel internacional para dar cuenta del turismo de naturaleza. El término se emplea en el ámbito americano principalmente, y es menos frecuente en el resto de los mercados. Engloba entre las vacaciones catalogadas como ecoturísticas las vacaciones de caminatas, las vacaciones de observación de aves, trekking en las montañas, trekking en el bosque pluvial y los safaris. Comprende estancias de hasta un día y abarca también el período temporal vacacional para disfrutar de áreas de notable belleza natural.

El turismo rural es una definición que ha sido muy debatida y cuestionada, y se le incluye en una actividad turística que se desarrolla en el medio rural, cuya motivación principal es la búsqueda de atractivos turísticos asociados al descanso, paisaje, cultura tradicional y huida de la masificación (Valdés Peláez, 1996, p. 376). En este sentido, la oferta turística es el conjunto de alojamientos, instalaciones, estructuras de ocio y recursos naturales y arquitectónicos existentes en zonas de economía predominantemente agrícola.

En las últimas corrientes del siglo XXI, el turismo alternativo se perfila como una definición más ajustada que abarca aquellos viajes cuya finalidad son 
las actividades recreativas en contacto directo con la naturaleza y con expresiones culturales que atañen al conocimiento, disfrute y conservación de los recursos naturales y culturales. Es también un turismo responsable, justo y solidario, de bajo impacto ambiental y cultural, ramificado entre el ecoturismo, el turismo de aventuras y el turismo rural.

\section{La Carta del Turismo Sostenible en 1995}

La Carta del Turismo Sostenible (Conferencia Mundial del Turismo Sostenible, celebrada en Lanzarote, islas Canarias, en 1995) se asentó en los documentos de Manila, Sofía, La Haya, la Declaración Universal de Derechos Humanos y la Declaración de Río sobre el Medio Ambiente y Desarrollo, cuyos principios fundamentales ubicaban el desarrollo turístico en la sostenibilidad, desde una visión integral que incorporase en todo su ciclo y a largo plazo la viabilidad ecológica y económica, y la equidad ética y social de las comunidades locales.

Este hito marca un antes y un después en la infraestructura de un turismo responsable, que insta a los gobiernos, autoridades públicas, profesionales, asociaciones, instituciones públicas y privadas y a los turistas hacia unos principios y objetivos determinados por el desarrollo turístico sostenible. Define una política turística de carácter sostenible con acciones positivas y preventivas, que proteja las zonas vulnerables ambiental y culturalmente, las zonas degradadas por modelos turísticos obsoletos y de alto impacto, la promoción de formas alternativas de turismo coherente con los principios del desarrollo sostenible y el fomento de la diversificación de los productos turísticos.

\section{El Código Ético Mundial para el Turismo en 2009}

El Código Ético Mundial para el Turismo (CEMT) tiene la finalidad de orientar a los agentes interesados en el desarrollo del turismo, por lo que engloba a los gobiernos centrales y locales, a las comunidades autóctonas, al sector turístico y sus profesionales y a los visitantes nacionales e internacionales. Es un marco de referencia para el turismo responsable y sostenible, que se demandó en una resolución de la Asamblea General de la OMT, celebrada en Estambul en 1997. Su reconocimiento oficial por parte de la Asamblea General de las Naciones Unidas tuvo lugar el 21 de diciembre de 2001 (en virtud de la resolución A/ $\mathrm{RES} / 56 / 212$ ), y se alentó por parte de la OMT la promoción de un seguimiento efectivo de dicho Código.

En el Artículo 3 se recoge el turismo como factor de desarrollo sostenible en los dos puntos siguientes:
4) "Se concebirá la infraestructura y se programarán las actividades turísticas de forma que se proteja el patrimonio natural que constituyen los ecosistemas y la diversidad biológica, y que se preserven las especies en peligro de la fauna y de la flora silvestre. Los agentes del desarrollo turístico, y en particular los profesionales del sector, deben admitir que se impongan limitaciones a sus actividades cuando éstas se ejerzan en espacios particularmente vulnerables: regiones desérticas, polares o de alta montaña, litorales, selvas tropicales o zonas húmedas, que sean idóneos para la creación de parques naturales o reservas protegidas. Y 5) "El turismo de naturaleza y el ecoturismo se reconocen como formas de turismo particularmente enriquecedoras y valorizadoras, siempre que respeten el patrimonio natural y la población local y se ajusten a la capacidad de ocupación de los lugares turísticos" (Organización Mundial del Turismo, Código Ético Mundial para el Turismo. Por un Turismo responsable, p. 5).

\section{La Carta Europea de Turismo Sostenible en los Espa- cios Protegidos, 2007-2010}

En la sinergia de la Carta del Turismo Sostenible (1995), se consolida la Carta Europea de Turismo Sostenible en los Espacios Protegidos, publicada por primera vez por la "Féderation des Parcs naturels régionaux de France" en 1999, que se revisa y actualiza por la Federación EUROPARC en 2007 y 2010. El desarrollo sostenible se aplica aquí en los espacios protegidos de Europa tales como los parques nacionales, parques naturales, áreas de belleza natural extraordinaria y reservas de la biosfera que, en su conjunto, forman parte del denominado turismo de naturaleza. Contiene las prioridades de la Agenda 21, adoptada en la Cumbre de la Tierra de Río de Janeiro (1992), y las de la Unión Europea en su 60 Programa de Acción y Estrategia Medioambiental para el Desarrollo Sostenible. Los objetivos básicos de la Carta Europea para el Turismo Sostenible son:

"Acrecentar el conocimiento de los espacios protegidos de Europa como parte fundamental de nuestro patrimonio y aumentar el apoyo a su necesaria conservación para que puedan ser disfrutados por las generaciones presentes y futuras."

“Mejorar el desarrollo sostenible y la gestión turística de los espacios protegidos, teniendo en cuenta las necesidades del entorno, de la población local, de las empresas locales y de los visitantes" (Carta Europea de Turismo Sostenible en los Espacios Protegidos, p. 4). 


\section{La Carta Mundial de Turismo Sostenible +20, 2015}

La Carta Mundial de Turismo Sostenible +20 (Vitoria-Gasteiz, 26 y 27 de noviembre de 2015) recuerda y valida los principios anteriores, y acuerdan que el turismo debe convertirse en un instrumento de paz y tolerancia que preserve los tesoros actuales para las generaciones futuras y asegure la integridad del patrimonio común, material e inmaterial. Se pone énfasis en la compatibilidad del turismo con la conservación y protección del medio ambiente, el cambio climático, desarrollar la resiliencia, contribuir a la economía mundial, luchar contra la pobreza, y utilizar, entre otros, las TIC y los bienes y servicios locales.

\section{LOS RETOS DEL TURISMO DE NATURALEZA: HACIA EL PARADIGMA DE LA RESPONSABILIDAD Y SOSTENIBILIDAD}

Todo el sector turístico está implicado de manera directa con el problema medioambiental, sociocultural y económico. Pero no todas las actuaciones de transformación e introducción de actividades producen impactos negativos, ya que el turismo, en muchas áreas, ha contribuido de manera importante a la conservación de la biodiversidad. The International Ecoturism Society (TIES) señala en sus informes que existen 109 países con arrecifes de coral; en 90 de ellos, los arrecifes son dañados por anclas de transatlánticos y aguas residuales, por turistas que rompen trozos del coral, y por cosechas comerciales para vender a los turistas. En Dominica y otras islas del Caribe los turistas que usaron hoteles pequeños basados en la naturaleza gastaron dieciocho veces más que los pasajeros en cruceros cuando visitaron la isla. Y mientras que el $80 \%$ del dinero gastado en paquetes todo incluido se destina a las líneas aéreas, los hoteles y a otras compañías internacionales, los denominados Eco-hoteles contratan y compran localmente, y a veces contribuyen con hasta el 95\% del dinero a la economía local. (The International Ecotourism Society (TIES), Hoja informativa. Ecoturismo Global, pp. 1-3).

El perfil del ecoturista en Europa da cuenta de un viajero experimentado, con un nivel de ingresos alto, de edad madura, que informa a sus amigos en sus viajes, y suele ser la fuente de información turística más importante. A nivel mundial se identifican algunos lugares catalogados como turismo ecológico tales como las Islas Galápagos (Ecuador), el Parque Nacional Marino de Fernando de Noronha (Brasil), la Reserva Biológica Privada de Bosque Nuboso de Monteverde (Costa Rica), la Reserva Biológica Privada de la Organización para Estudios Tropicales (Costa
Rica), el Parque Nacional Kruger (Sudáfrica), el Parque de la Reserva Maasai Mara (Kenia) y el Parque Nacional Turuepano (Venezuela). Todos ellos atraen importantes cantidades de turistas y son objeto de inevitables impactos negativos.

Para las comunidades locales pobres el ecoturismo representa una salida fundamental a las limitadas posibilidades en su modo de vida, y favorece el mantenimiento y la salida del círculo de la pobreza. Asimismo, es esencial la gestión y la protección de los ecosistemas del mundo, para que no sean presa de la deforestación, la agricultura excesiva y el urbanismo masificado. La organización de UK Tourism Concern, vigila y demanda campañas de organizaciones en países de destino, que han aplicado el ecoturismo y han impactado negativamente sobre el medioambiente, las personas y las culturas.

Para que funcione el ecoturismo es necesario que además de proveer buenas medidas de conservación, la comunidad tenga posibilidades significativas de participación, como es el caso de Namibia, país cuyo turismo se basa en la comunidad y en los safaris, y dispone de una amplia diversidad de grupos étnicos que se han implicado en el turismo (Tejera, J. Africa Scientia 3/4: Turismo responsable en Namibia y Lesoto). Aquí se han establecido campamentos apoyados por iniciativas privadas y públicas. La Asociación Namibia de Turismo con Base Comunitaria representa y promueve veintiún campamentos comunitarios, museos y negocios de giras. Los beneficios del turismo se dividen entre las comunidades, y el turismo es manejado por ellos para su beneficio.

También es muy significativo el caso de Ecuador con Yachana Lodge (www.yachana.com), asentamiento que fue construida por la comunidad Quichua de Mondana. En esta localidad los visitantes pescan pirañas, nadan en el río Napo y caminan a través de la Amazonía. Los ingresos de las cabañas son los que ayudan a financiar una clínica de salud, una granja apícola y de permacultura ${ }^{1}$.

Entre los retos del turismo de naturaleza sobresalen el deterioro medioambiental, las catástrofes naturales y el cambio climático, que corresponden a la dimensión de los impactos procedentes de factores internos y externos a la industria turística y del ocio. El Código Ético Mundial para el Turismo da cuenta del valor incalculable que tiene el medio ambiente y los recursos naturales, y en el artículo 3 especifica que "Todos los agentes del desarrollo turístico tienen el deber de salvaguardar el medio ambien- 
te y los recursos naturales, en la perspectiva de un crecimiento económico saneado, constante y sostenible, que sea capaz de satisfacer equitativamente las necesidades y aspiraciones de las generaciones presentes y futuras" (Organización Mundial del Turismo. Código Ético Mundial para el Turismo. Por un Turismo responsable, pp. 4-5).

El turismo responsable va tomando conciencia de una serie de valores y de una gestión que tenga en cuenta la fragilidad del medio ambiente, y coexista en equilibrio con las industrias turísticas, como es el caso de la observación de aves (Wikipedia). Ficapal (2007) está interesado en el desarrollo que representa el bienestar de las personas y las comunidades a través de la satisfacción de sus necesidades. Y Benito Fraile (2007) se preocupa por la conservación de los recursos medioambientales, económicos y socioculturales que existen en los destinos turísticos. Aquí subyacen, pues, elementos esenciales de responsabilidad social empresarial (responsabilidad económica, legal, ética y altruismo-compromiso con la sociedad).

La OMT ya vislumbró en el Código Ético Mundial para el Turismo, este modelo de desarrollo económico que se organiza para mejorar la calidad de vida de la población local, la del medio ambiente y la de los visitantes, sin menoscabar la rentabilidad económica de los residentes locales y la de los empresarios turísticos. En este sentido, ha de procurarse que el negocio turístico sustentable sea rentable, porque de lo contrario los empresarios olvidarán el compromiso de sustentabilidad y podrían alterar el equilibrio (Sancho y Buhalis, 1998, p. 260).

\section{LA PERCEPCIÓN DE LOS PAISAJES CULTURALES COMO PRODUCTOS TURÍSTICOS: UNA PUESTA EN VALOR DE LA NATURALEZA}

La visualización de la naturaleza a través del turismo lleva al conocimiento no solo del medio natural sino también de los rasgos culturales de sus poblaciones y la sensibilización de implicación en la conservación de los mismos. Una de las tareas de la planificación consiste en satisfacer las necesidades de interpretación y de información de los visitantes y la gestión que comporta, y se requiere proporcionar una educación mínima, dado que los turistas desean aprender acerca de los ambientes que visitan y conseguir una mejor comprensión y apreciación del ambiente natural-cultural. De aquí que nos centremos ahora en conocer la percepción de los paisajes culturales como productos turísticos en los turistas que los frecuentan.
Los paisajes culturales se definen como el resultado de la interacción de las personas con su medio natural a través del tiempo, y constituyen un territorio que es percibido y valorado según sus cualidades culturales, pertenecientes a un proceso y soporte de identidad de una comunidad (Plan Nacional de Paisaje Cultural). Son, por tanto, sitios y lugares de especial valor natural y cultural, de carácter histórico, que según los análisis de Zárate Martín (2012) están comprendidos muy a menudo en ámbitos urbanos o bajo la influencia de las ciudades en entornos paisajísticos de gran calidad y singularidad que se conforman en símbolos de identificación social y en recursos susceptibles de generar riqueza, a condición de mantenerlos y conservarlos desde criterios de sostenibilidad y de protección del patrimonio.

Las nuevas tendencias del turismo de naturaleza incorporan los paisajes culturales en paralelo a los procesos de conservación del paisaje en el urbanismo y la ordenación del territorio. Prueba de ello son la Carta Internacional para la Conservación de las Ciudades Históricas y las Áreas Urbanas de 1987 (Carta de Toledo-Washington), la Carta Internacional sobre Turismo Cultural y la Convención Europea del Paisaje del año 2000 (Florencia), que representan una defensa de los paisajes y los valores patrimoniales a través del turismo.

Los paisajes culturales como nuevo producto turístico dan cuenta de los recursos históricos y artísticos con sus entornos naturales y paisajísticos y entran en la modalidad de las nuevas demandas en ocio y turismo en la actualidad. La cultura es el núcleo central, y sus estancias oscilan entre los tres y los cuatro días, siendo las ciudades históricas la fuente de atracción principal con todos los monumentos, paisajes, urbanismo reconstruido y áreas de ocio y recreación colindantes a ellas.

La percepción de los paisajes culturales como producto turístico que aquí se expone se ha realizado a través de una investigación cuantitativa (CULTURPAIS, $2015)^{2}$ mediante encuesta ${ }^{3}$, con un cuestionario de preguntas abiertas, cerradas y mixtas, y de escala, que sirve como introducción y contribución en la valoración que tienen los turistas sobre este nuevo producto. Entre las respuestas más significativas a la pregunta sobre la duración de la visita a ciudades históricas ricas en paisajes, el $26,1 \%$ contestó que hacía la ida y la vuelta en el mismo día, el 27,2\% permanecía más de dos noches, el 19,1\% dos noches, el 9,6\% sólo una noche y el $17,8 \%$ hacía estas visitas coincidiendo con la estancia vacacional. Las visitas se suelen realizar 
principalmente los fines de semana, en vacaciones de verano y entre semana, y suelen acudir mayoritariamente acompañados, en familia, en grupos de amigos y conocidos.

En la tabla 1, obtenida mediante un análisis básico de comparación de medias, figuran las motivaciones de la visita a ciudades con entornos paisajísticos y culturales que están determinados por el interés de recorrer las calles y plazas del lugar $(4,26)$, visitar monumentos $(4,12)$ y contemplar varias vistas de la localidad $(4,06)$. Actividades en su conjunto que requieren desplazamiento físico y sin un consumo turístico excesivo. En general, los turistas que respondieron a la encuesta muestran satisfacción global de la visita $(4,46)$, y en la contemplación del paisaje cultural prefieren las vistas del conjunto de la ciudad y de su casco histórico $(4,45)$, y las vistas y paisajes de su naturaleza $(4,04)$.

Respecto a los factores de calidad de los paisajes culturales los turistas señalan la importancia de la limpieza en los lugares y entornos $(4,13)$, la indicación de vistas panorámicas $(4,2)$, la existencia de lugares para poder tener buenas vistas $(4,09)$, que estén en buen estado de conservación $(3,98)$ y de fácil acceso con aparcamientos (3,32). Por último, indican la importancia que tienen las vistas del conjunto histórico desde lo alto de una torre o de cualquier otro edificio $(4,23)$, las vistas de espacios interiores tales como plazas, calles, cruce de calles $(4,18)$ y las vistas desde lugares donde existan buenas panorámicas $(4,11)$.

En general, en el interés del turista subyace un orden en la captación y disfrute de la visita, es decir, primero se quiere conocer y disfrutar la totalidad del lugar a través de las panorámicas más generales, a modo de vista general, para pasar después a lugares y actividades más concretas como visitar monumentos y ver museos, y realizar al final de la visita recorridos comentados por las diversas vistas de la localidad. Las mujeres son las que puntúan más alto la preferencia de las vistas panorámicas de la ciudad, las de monumentos y museos y los recorridos comentados por las diversas vistas, en tanto que los hombres puntúan en una menor proporción esta última opción.

Según género, grupo de edad y nivel de estudios se plasman ciertas preferencias por dos tipos de paisajes culturales. El paisaje cultural urbano e histórico es valorado más por los hombres jóvenes-adultos y por los

Tabla 1. Percepción de los turistas sobre los paisajes culturales como productos turísticos

\begin{tabular}{|c|c|}
\hline MOTIVACIONES DE LA VISITA & $\bar{X}$ \\
\hline -Recorrer calles y plazas del lugar. & 4,26 \\
\hline -Visitar monumentos. & 4,12 \\
\hline -Contemplar varias vistas de la localidad. & 4,06 \\
\hline SATISFACCIÓN EN LA CONTEMPLACIÓN DEL PAISAJE CULTURAL & $\bar{X}$ \\
\hline -Satisfacción global de la visita. & 4,46 \\
\hline -Vistas del conjunto de la ciudad y de su interior. & 4,45 \\
\hline -Vistas y paisajes de su naturaleza. & 4,04 \\
\hline FACTORES DE CALIDAD DE LOS PAISAJES CULTURALES & $\bar{X}$ \\
\hline -Limpieza en los lugares y entorno. & 4,13 \\
\hline -Indicación de vistas panorámicas. & 4,2 \\
\hline -Existencia de lugares para poder tener buenas vistas. & 4,09 \\
\hline -Estar en buen estado de conservación sin elementos que los perturben. & 3,98 \\
\hline -Facilidades de acceso y aparcamiento. & 3,32 \\
\hline IMPORTANCIA DE LAS VISTAS PANORÁMICAS Y ESCENARIOS URBANOS & $\bar{X}$ \\
\hline -Vistas del conjunto desde lo alto de una torre o de cualquier otro edificio. & 4,23 \\
\hline -Vistas de espacios interiores (plazas, calles, cruce de calles). & 4,18 \\
\hline -Vistas desde lugares donde existan buenas panorámicas. & 4,11 \\
\hline
\end{tabular}

Fuente: Investigación CULTURPAIS 2015. 
adultos con estudios universitarios y secundarios que utilizan medios tecnológicos divulgativos tales como las lecturas, la prensa e Internet. En cambio, el paisaje cultural natural y panorámico es preferido por las mujeres, sobre todo de edades intermedias y avanzadas, con formación profesional y estudios primarios, que obtienen información de los lugares que van a visitar a través de los amigos, familiares y conocidos principalmente. Finamente, en el análisis DAFO de los paisajes culturales como producto turístico se destacan los siguientes resultados:

\section{FORTALEZAS DE LOS PAISAJES CULTURALES}

Los paisajes culturales son visitados de dos a cinco veces en la vida como media.

Este perfil de turista cultural es comunitario, por lo que el acompañamiento va a ser uno de los elementos claves que conforman grupos de familias, amigos y conocidos en esta modalidad turística.

Recursos naturales con vistas panorámicas únicas en ciudades históricas únicas.

Hay satisfacción con la contemplación del paisaje cultural, seguida del comportamiento recreativo social y consumista locales.

Los elementos de calidad de los paisajes culturales están bien aprovechados para las vistas panorámicas y los entornos naturales.

Hay un modelo de turista cultural ajustado a la oferta del paisaje cultural: a) paisaje cultural urbano e histórico para los hombres, b) paisaje cultural y panorámico para las mujeres.

\section{DEBILIDADES DE LOS PAISAJES CULTURALES}

Hay escasez de imágenes en la web de cara a la atracción turística.

No conocer las necesidades de los visitantes motivados por el turismo cultural como valor intangible fundamental.

Tener patrimonio no asegura ser un destino patrimonial en sí mismo.

No disponer de buenas guías turísticas.

Mejorar los accesos y aparcamientos, la conservación y elementos visuales que perturban las vistas panorámicas, señalizar mejor las rutas de los espacios urbanos y naturales, y finalmente habilitar más espacios o lugares desde donde poder tener esas vistas. Las infraestructuras están todavía poco adaptadas a la sostenibilidad y a la capacidad de carga.

\section{OPORTUNIDADES DE LOS PAISAJES CULTURALES}

Son fuente de riqueza cultural y educativa para lo local, nacional, regional e internacional.

Se integra el patrimonio local, el turismo y las rutas culturales.

Es una alternativa al desarrollo turístico, es turismo alternativo.

Las actividades al aire libre (recorrer calles y plazas, visitar monumentos, contemplar vistas de la localidad) requieren desplazamiento físico y no tienen un consumo turístico excesivo, incluso a veces es muy reducido o prácticamente nulo.

Más de tres cuartas partes de los turistas de la investigación recomendarían el lugar visitado y considerarían las vistas como destino cultural.

\section{AMENAZAS DE LOS PAISAJES CULTURALES}

Existe fuerte competencia de otras ciudades con marca Ciudad Patrimonio de la Humanidad con mejores equipamientos.

Falta de planificación urbanística turística de cara a la afluencia consolidada de este turista que no tiene estacionalidad, es constante en el tiempo.

Falta acondicionamiento de infraestructuras para las personas con diversidad funcional.

\section{PROPUESTA DE PLANIFICACIÓN DEL TURISMO DE NA- TURALEZA COMO PRODUCTO TURÍSTICO SOSTENIBLE}

Sin duda el modelo de turismo responsable y sostenible ha frenado, en cierta medida, la devastación casi imparable que había supuesto el modelo de crecimiento expansivo convencional que ejerció una importante degradación de los recursos naturales. Especialistas como Grifols (2003), Pulido Fernández (2003) y Boyra Amposta (2007) se han encaminado a través de sus perspectivas en este modelo que ahora presentamos.

Así, por ejemplo, Grifols (2003, p. 95) explica que debe reunir una serie de características imprescindibles, como la de ser un turismo ecológicamente aceptable, respetuoso con el entorno, que reduzca los impactos negativos y socialmente justo para que la población local se beneficie y potencie sus valores. Deberá integrar, diversificar y adaptarse a la personalidad del sitio, por mediación de empresas locales y por la participación de todos los actores en el lugar de destino.

Este modelo confluyó con el movimiento verde de los países desarrollados, las denuncias de los especia- 
listas y las presiones de los medios de comunicación, para cambiar la legislación existente en la Unión Europea, y para que la población y las empresas tomasen conciencia de la situación. Pero el proceso no fue el mismo en los países en vías de desarrollo que se encontraban en otras fases de desarrollo y despegue de la industria del turismo. Por lo que se produce una disfuncionalidad en el sector turístico que se ha ido implantando a distintas velocidades.

Pulido Fernández (2003) recoge términos relacionados con la sostenibilidad y el turismo cercano al hábitat de la naturaleza, en un momento en que se utilizan indistintamente expresiones como: turismo verde, turismo de naturaleza, turismo en espacios naturales protegidos, ecoturismo, turismo ecológico, agroturismo, turismo de aventura, turismo activo, turismo alternativo, turismo sostenible, turismo sustentable, turismo blando o turismo de impacto ambiental. Una de las confusiones halladas ha sido la de asociar el ecoturismo con el de turismo sostenible, siendo este último mucho más global por abarcar cualquier tipología de turismo, e incluso se confunde con la modalidad del turismo de naturaleza, que se basa en elegir la naturaleza principalmente, y se constituye en la motivación principal del viaje para el disfrute del ocio y tiempo libre.

Boyra Amposta (2007) profundiza en el nuevo paradigma que se plantea con el concepto de sostenibilidad de cara a la planificación, que versa sobre la búsqueda del equilibrio entre desarrollo y conservación. La industria del turismo y las Administraciones del Estado deben afrontar los nuevos retos con la promoción y el desarrollo cuantitativo, así como la competitividad por la vía de la calidad y la eficacia. Se requieren fórmulas de gestión pública innovadoras, que apoyen la conformación de entornos favorables y enclaves turísticos sostenibles. En este modelo resulta imprescindible la aplicación de métodos y técnicas concretos sobre indicadores de calidad medioambiental destinados a zonas turísticas sostenibles, con el fin de asegurar la competitividad según los principios de sostenibilidad.

En esta corriente se aplican indicadores específicos para los destinos turísticos elaborados por Ayuso y FuIlana (2001), que se agrupan en diez principalmente:

- Protección del lugar. Medición: categoría de protección del lugar según el índice de la Unión Internacional para la Conservación de la Naturaleza (IUCN).

- Presión. Número de turistas que visitan el lugar (por año y mes).
- Intensidad de uso. Temporada alta (personas/hectáreas).

- Impacto social. Proporción de turistas respecto de los residentes locales (temporada alta).

- Control del desarrollo. Existencia de procedimientos de revisión ambiental o controles oficiales de desarrollo del lugar y de intensidad de uso.

- Tratamiento de residuos. Porcentaje de aguas residuales que se someten a tratamiento (también pueden tomarse como indicadores los límites estructurales de otras capacidades infraestructurales del lugar: por ejemplo, suministro de aguas, basuras).

- Proceso de planificación. Existencia de un plan regional organizado por la región del destino turístico.

- Ecosistemas críticos. Número de especies poco frecuentes o en peligro.

- Satisfacción del consumidor. Grado de satisfacción de los visitantes (basado en un cuestionario).

- Satisfacción de la población local. Grado de satisfacción de la población local (basado en un cuestionario).

Los indicadores medioambientales ofrecen una información previa del estado de salubridad y viabilidad en la que se encuentran los destinos turísticos y son básicos para la planificación. La OMT ofreció dos tipos de indicadores: a) indicadores básicos de turismo sostenible que se pueden aplicar a todos los destinos (protección del sitio, presión, intensidad de uso, impacto social, existencia de procedimientos de revisión ambiental o controles oficiales de desarrollo del sitio y densidades de uso, tratamiento de desechos, proceso de planificación, ecosistemas críticos, satisfacción del consumidor, satisfacción de la población local) y b) indicadores específicos de destinos para ser aplicados a ecosistemas o tipos de turismo concretos como zonas costeras, regiones montañosas, parques naturales, sitios culturales, comunidades tradicionales y pequeñas islas. (Sancho y Buhalis, 1998, pp. 269-270).

Getz (1983) aglutinó tres fases en el proceso de planificación: 1) formulación de un modelo de priorización de objetivos, 2) evaluación de las estrategias y 3) selección de procedimientos. En la priorización de objetivos se identifican los problemas, de manera que se puedan conseguir con la aceptación armoniosa de las autoridades locales, la población receptora, las em- 
presas y los visitantes, lo que lleva a la coordinación del sector público y privado en los ámbitos nacionales, regionales y locales.

Para Rivas García y Magadán Díaz (2008) la planificación estratégica del turismo implica elaborar un proyecto que integre el área geográfica, para convertirla en un producto turístico que comprende un conjunto de elementos como el paisaje, las empresas, las infraestructuras, los equipamientos, el entorno social, el patrimonio, etc. Los objetivos serán específicos para cada gobierno, conforme a las expectativas de crecimiento y desarrollo, en aras de la promoción del tipo de turismo deseable, con estadísticas fiables y la coordinación con el resto de políticas.

La planificación correcta plantea un análisis de antecedentes, que corresponde a la observación previa a la planificación y desarrollo; a partir de ahí se establece una oferta turística que abarca el inventario, las infraestructuras, los recursos turísticos efectivos, los recursos turísticos potenciales y la encuesta sectorial. En el análisis de la demanda se consideran la información de lo que desean los turistas, cómo vienen, cuánto tiempo, qué tipo de alojamiento, qué gastan, etc. Sancho y Buhalis (1998, pp. 187-188) exponen que el término Plan de Desarrollo Turístico se ha empleado haciendo referencia a la planificación turística y es un plan estratégico que integra todos los aspectos del desarrollo turístico entre los que se incluyen los recursos humanos, los medioambientales y los socioculturales.

En definitiva, la propuesta de planificación del turismo de naturaleza como producto turístico sostenible trabaja con seis agentes principales: los gobiernos, las empresas privadas, las comunidades locales, la industria turística, los turistas y las organizaciones no gubernamentales (ONG). Entre las acciones que deben realizar los gobiernos a favor del desarrollo del turismo sostenible figuran el trabajo conjunto con los empresarios en el establecimiento de políticas sustentables, que proporcionen una política de incentivos para favorecer el crecimiento equilibrado. Por tanto, será necesario un programa de evaluación de impactos sobre los destinos turísticos, un control de la capacidad de carga de los mismos y la creación de auditorías de calidad ambiental para incluir el turismo en los planes de gobierno.

El papel de las comunidades locales consiste en proporcionar interacciones culturales entre la comunidad local y los visitantes, proporcionar servicios, potenciar los productos locales, tomar decisiones en los diseños de los proyectos e iniciativas respecto a las acciones, participar con los costes de los proyectos y proteger las normas culturales. A su vez, la industria turística contribuye para que se elimine el uso de herbicidas y exista un desarrollo equilibrado del uso del terreno, el agua y los bosques. Del mismo modo, debe efectuar un buen tratamiento de los residuos sólidos y líquidos, adoptar técnicas energéticas eficientes, prácticas de marketing verde, y minimizar los riesgos de intoxicaciones (guía o información diversa a los turistas) para orientar en las actitudes y comportamientos responsables e incorporar los valores del medioambiente en los procesos de decisión empresarial y auditorias propias medioambientales.

Con respecto a los turistas, las acciones se encaminan hacia la tendencia de saber seleccionar los destinos con responsabilidad medioambiental y conocer los mecanismos de integración en las comunidades autóctonas, para llevar a cabo actividades con escaso impacto y de conservación del medio ambiente. Las ONG pueden intervenir también en la participación de los comités de control medioambiental, en la creación de acciones de apoyo al desarrollo sostenible, y en el control de los impactos de comunidades locales.

En España, en la propuesta de planificación del turismo de naturaleza se incorporan las principales directrices del Plan Sectorial de turismo de naturaleza y biodiversidad 2014-2020 (Boletín Oficial del Estado núm. 147 , de 18 de junio de 2014), que parte de un concepto amplio que engloba un gran número de actividades que se pueden realizar en el medio natural como entorno principal, en el que están directamente relacionados el ecoturismo, el turismo activo o el turismo de esparcimiento. La perspectiva económica que ofrece es excelente ya que reúne unos requisitos óptimos para generar beneficios en las economías locales, y una oportunidad de desarrollo económico y social basado en los recursos naturales procedentes del territorio.

La confluencia entre la conservación del medio natural y la planificación y promoción del turismo llevan a un desenvolvimiento de economía expansiva y sostenible a largo plazo. España ocupa una posición privilegiada para este turismo en el nivel nacional y en el contexto europeo. Pone a disposición productos y destinos calificados como de exclusivos, diferenciadores y de calidad, que se unen al atractivo de paisajes y modos de vida tradicionales, y que atrae a muchos turistas y visitantes. La viabilidad de este turismo viene determinada por la conservación, el mantenimiento y la mejora de la biodiversidad, y la planificación y la ubicación de las actividades son las que dan garantía y seguridad de su sostenibilidad. 
La participación de la sociedad y la colaboración entre los agentes son totalmente necesarios, y deben estar informados, sensibilizados y concienciados sobre los principios y valores del medio ambiente, así como los problemas que hay que afrontar, y conocer las repercusiones de las actividades en el medio para minimizar el impacto e impedir que haya efectos negativos.

En la tabla 2 la propuesta de planificación del turismo de naturaleza como producto turístico sostenible está compuesta de tres fases. La observación y estudio previo del medio es la primera fase, que abarca la oferta, la demanda y los indicadores. La oferta se encarga de hacer un inventario, conocer las infraestructuras y recursos y potenciarlos, y se utiliza una encuesta sectorial. La demanda recoge la información de lo que desean los turistas y las necesidades que tiene la población autóctona.

Con el diagnóstico disponible la segunda fase pasa a la planificación cooperativa entre los seis agentes (gobierno, empresas privadas, comunidades locales, industrias turísticas, turistas y ONG) para diseñar los objetivos de desarrollo del destino en un plan estratégico y con políticas turísticas. La elaboración de una imagen del destino es fundamental, y se aplican las técnicas avanzadas del marketing turístico digital. $Y$ en su conjunto toda la planificación cooperativa debe aplicar las normativas locales, nacionales e internacionales con los principios y valores del modelo de turismo responsable y sostenible.

Por último, la tercera fase requiere la consideración y el reconocimiento del producto turístico sostenible, que se dirige al núcleo central del modelo, a través de la conservación y protección del medio ambiente. Para conseguir este producto los agentes y gestores tienen que asumir un compromiso y asegurar la compatibilidad con los objetivos de conservación de los valores naturales del espacio. Finamente es un requisito imprescindible mejorar los conocimientos, la información y la formación del turismo de naturaleza en general, y la de los paisajes culturales en particular.

\section{CONCLUSIONES}

El turismo de naturaleza es la "frontera" de todos los turismos, y el punto inicial y final en los procesos experimentales del sector turístico. La materia principal es el patrimonio natural- cultural, y todos los beneficios y riquezas que se obtengan marcarán el éxito o el fracaso de esta industria. Si se destruye el medio ambiente y se altera la autenticidad de los destinos turísticos, dispondremos de espacios desgastados, deshabitados y desestructurados, en los que la experiencia del disfrute del viaje y de la estancia pierda su significado y razón de ser.

Tabla 2. Propuesta de planificación del Turismo de Naturaleza como producto turístico sostenible

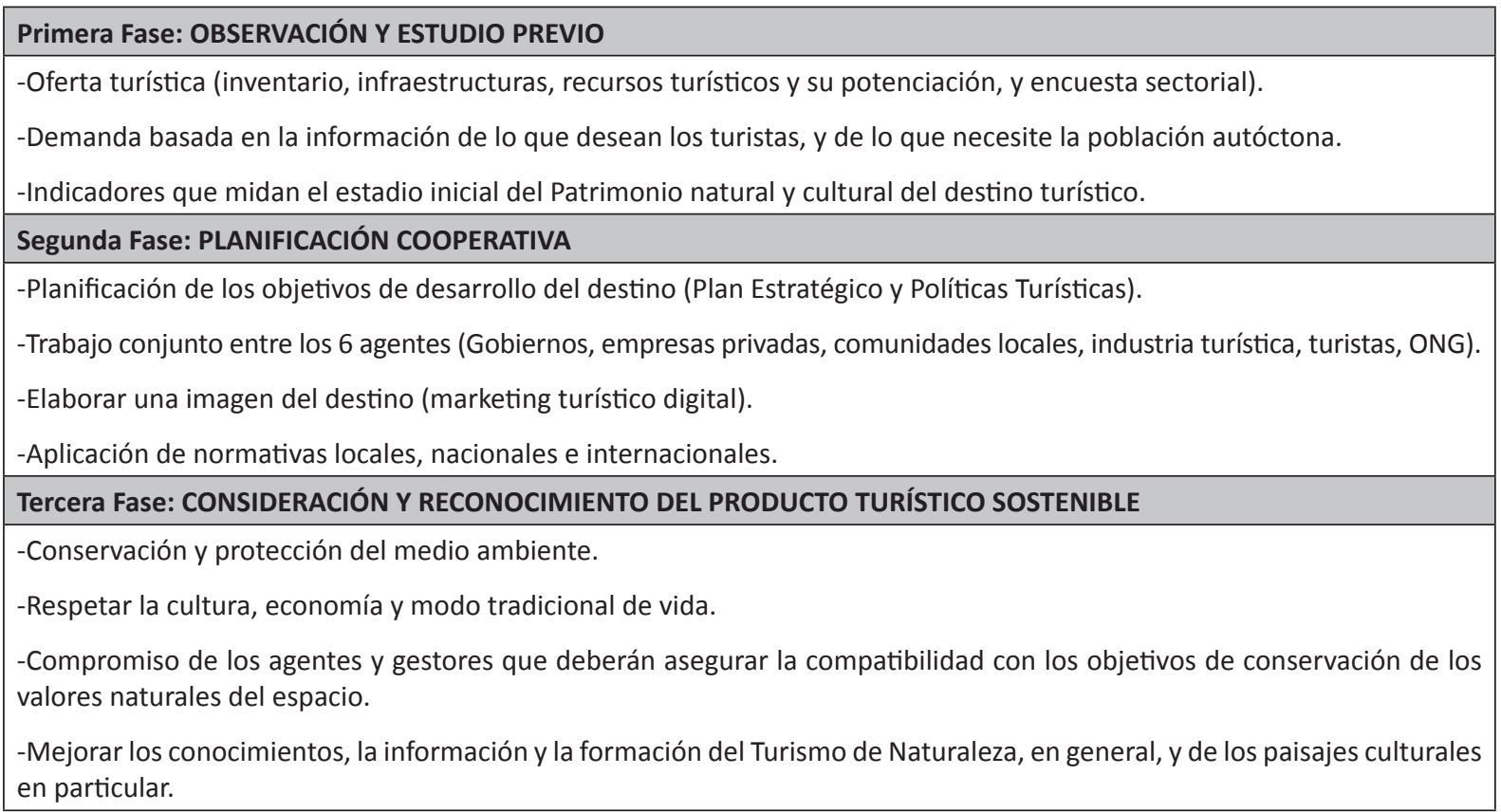

Fuente: elaboración propia según varios autores y el Plan Sectorial de Turismo de Naturaleza y Biodiversidad 2014-2020. 
Los hábitats naturales y su biodiversidad se han puesto en alza en el mercado turístico y se han mercantilizado todos los recursos y potencialidades, desde un paseo por senderos ricos en flora y fauna, hasta los deportes de aventuras en globo, pasando por la gastronomía más sabrosa frente a paisajes paradisíacos y únicos. Más allá de estas "fronteras" no hay nada, por lo que el camino de retorno requiere la recuperación, el respeto y la valorización del patrimonio natural-cultural, y llegar a conseguir de la naturaleza y su disfrute un producto turístico sostenible.

Tal y como se ha visto en los resultados del proceso turístico, los impactos negativos del modelo convencional le han hecho caer por su propio peso, y se ha visto obligado a cambiar el mecanismo de desarrollo expansivo y abrasivo por el desarrollo local sostenible. Es un hecho ya la implantación progresiva del paradigma de la responsabilidad y sostenibilidad en los destinos turísticos asentados en los entornos naturales.

En consecuencia, tanto los organismos públicos como los privados, se han igualado en ajustar el turismo de naturaleza en los principios de sostenibilidad. La OMT, las Naciones Unidas y los Gobiernos han ido poniendo la voz de alarma a una industria insostenible que ha llevado al desgaste, la destrucción y la alteración de los espacios naturales-culturales y su autenticidad, modificando la interacción social y los significados sociales. $Y$ han sido los retos que han planteado todos los turismos los que han desembocado en el turismo de naturaleza, los que han dado paso al agotamiento del modelo convencional por el inicio y emprendimiento del modelo alternativo responsable y sostenible, haciendo un uso equilibrado del mismo de manera obligada por la fuerza de los hechos.

Todas estas conclusiones y propuestas a las que se llega son consecuencia de los análisis de las iniciativas para el fomento de un turismo de naturaleza alternativo, que tiene como base la conservación de los recursos, la recuperación, el respeto y la valorización. Por tanto, la comercialización, el disfrute y la expansión económica se asientan en la preservación del medio sin alterarlo y modificarlo. Todo ello implica un esfuerzo de restauración a corto y medio plazo, que tiene como eje principal la política de conservación del medio natural y la planificación y promoción del turismo.

El uso de los recursos naturales requiere evaluación, conocimiento, contribución al entendimiento y resolución de los problemas que afectan a la calidad de vida (Macpherson Mayol, 2000), todos ellos entran en el nuevo modelo alternativo. Por ello, la sociedad, el tiempo y el espacio de esa posmodernidad que se caracteriza por una intensa degradación del medio ambiente, que Aledo Tur (2008) perfila en sus estudios sobre la transformación del paisaje por el turismo, ahora se torna en lo contrario, en una vuelta de tuerca y de ajuste hacia la restauración, el respeto y la valoración. En consecuencia, los referentes modernos del siglo XXI están encaminados a reactivar con mucha velocidad los procesos de identificación social e individual hacia estos principios y valores sostenibles, que pongan en su sitio los esquemas básicos, las actitudes y experiencias en los fenómenos socioculturales que emanan del turismo, y sobre los elementos más tangibles de los destinos turísticos, de los tiempos de ocio y del consumo de los mismos.

El tiempo de la globalización de un mundo amenazado por las incertidumbres, los riesgos y la degradación medioambiental habría terminado, y daría paso a otro nuevo orden en el que lo local cobra protagonismo, y el desarrollo sostenible es obligado. En este sentido, las nuevas identidades y significados sociales han ido por delante de los proyectos, planes y políticas turísticas a implementar, y ahora corresponde plasmarlos en las nuevas realidades turísticas que, como en el caso de los paisajes culturales, perciben el patrimonio natural-cultural con satisfacción en general, y demandan buen estado de conservación, conocimiento y mejora de la formación para su contemplación y disfrute.

Hay una vuelta a la autenticidad de los valores patrimoniales como plazas, calles, monumentos, poblaciones y sus culturas, lugares, panorámicas y paisajes, así como al interés de conocerlos in situ y experimentarlos en compañía de la familia, amigos y conocidos, sin un consumo excesivo, es el denominado modelo responsable y sostenible que se impone como el mejor de los caminos. Todo ello está inserto desde hace tiempo en la Carta del Turismo Sostenible, el Código Ético Mundial para el Turismo (OMT), la Carta Europea de Turismo Sostenible en los Espacios Protegidos y en los diecisiete Objetivos de Desarrollo Sostenible de Naciones Unidas, con su Agenda 2030.

Por último, la propuesta de planificación basada en este modelo de turismo responsable y sostenible constituye un punto de arranque, que tiene como objetivo principal garantizar la conservación y sostenibilidad del patrimonio natural-cultural como producto turístico sostenible. Al igual que otras propuestas llevadas a cabo como las de Noguera Tur, Ferrándis Martínez y Riera Spiegelhalder (2012), que están orientadas a impulsar la recuperación y puesta en valor del 
patrimonio cultural con la finalidad de poder disfrutar la sociedad local, y mejorar el sentimiento de identidad en el propio territorio y cultura, con un Plan de Acción de actuaciones programadas.

La capacidad de éxito de la propuesta radica en la probabilidad de alcanzar los objetivos en sus tres fases, la de observación y estudio previo de la oferta y la demanda, y los indicadores que miden el estado inicial del patrimonio natural-cultural. Una segunda fase centrada en la planificación de cooperación entre los seis agentes con planes estratégicos y políticas turísticas, y una tercera fase de consideración y reconocimiento del producto turístico sostenible, que se comprometa en la promoción del turismo de conservación y protección, asegurando la calidad y proporcionando una mejora en los conocimientos, información y formación del turismo de naturaleza.

De aquí al 2030 la propuesta de alcanzar un producto turístico sostenible debe ser compatible con el escenario de fondo posmoderno que trazan los diecisiete Objetivos de Desarrollo Sostenible: fin de la pobreza, hambre cero, salud y bienestar, educación de calidad, igualdad de género, agua limpia y saneamiento, energía asequible y no contaminante, trabajo decente y crecimiento económico, industria, innovación e infraestructura, reducción de las desigualdades, ciudades y comunidades sostenibles, producción y consumo responsables, acción por el clima, vida submarina, vida de ecosistemas terrestres, paz, justicia e instituciones sólidas y alianzas para lograr los objetivos.

\section{NOTAS}

1. Es un sistema de principios de diseño agrícola y social, político y económico basado en los patrones y en las características del ecosistema natural. La trayectoria de la permacultura empieza con la ética y los principios de diseño y se mueve a través de etapas claves necesarias para crear un futuro sostenible. Estas etapas están conectadas por un camino evolutivo en forma de espiral, inicialmente en un nivel personal y local, para después proceder a lo colectivo y global (https://permacultureprinciples.com/ es/pc_flower_poster_es.pdf).

\section{BIBLIOGRAFÍA}

Aledo Tur, A. (2008). De la tierra al suelo: la transformación del paisaje y el nuevo turismo residencial. Arbor, 729, pp. 99-113. https://doi.org/10.3989/arbor.2008. i729.164

Ayuso, S. y Fullana, P. (2001). Turisme Sostenible. Barcelona: Generalitat de Catalunya.

Benito Fraile, A. E. (2007). Del Turismo Sostenible al Turismo Socialmente Responsable. En: Desafíos y compromisos del turismo: hacia una visión más humana. Congreso UNIJES. Bilbao: Universidad de Deusto, pp. 333-345.

Boyra Amposta, J. (2007). Turismo y Desarrollo: los términos de una dialéctica. En: Desafíos y compromisos del turismo: hacia una visión más humana. Congreso UNIJES. Bilbao: Universidad de Deusto, pp. 177-203.
2. Investigación sobre Percepción y valoración del Paisaje Cultural como producto turístico 2014, dentro del proyecto "Dinámicas comparadas para una gestión sostenible de los paisajes culturales a través del turismo" 2015 (CULTURPAIS), Ref. CSO2011-24966, Ministerio de Ciencia e Innovación. Investigador principal: M. Antonio Zárate Martín. Se han utilizado las técnicas descriptivas univariables (frecuencia y medias) y un análisis DAFO para conocer la situación real a través de las conclusiones.
Chafe, Z. (2005). Interest in Responsible Travel Grows. En: Vital Signs 2005. New York-London: W.W. Norton \& Company, p. 100-101.

Comisión Mundial del Medio Ambiente y del Desarrollo y Brundtland, G. H. (1989). Nuestro Futuro Común. Disponible en: https://es.scribd.com/doc/105305734/ ONU-Informe-Brundtland-Ago-1987-Informe-de-la-Comision-Mundial-sobreMedio-Ambiente-y-Desarrollo.

Epler Wood, M. (2002). Ecotourism: principles, practices \& policies for sustainability. Paris: United Nations Enviroment Programme (UNEP).

Ficapal, J. (2007). Turismo responsable, ¿pasaporte al desarrollo? En: Desafíos y compromisos del turismo: hacia una visión más humana. Congreso UNIJES. Bilbao: Universidad de Deusto, pp. 39-45.
3. La encuesta se obtuvo mediante la realización de un total de 500 encuestas telefónicas realizadas dentro del ámbito, según el cuestionario preestablecido. El trabajo de campo se realizó en abril y mayo de 2014 (error muestral + - $-4,47 \%$, bajo el supuesto de muestreo probabilístico, nivel de confianza 95,5\%; $p=q=0,5$, procedimiento de muestra aleatorio simple) distribuido por comunidades Autónomas en España.
Getz, D. (1983). Capacity to absorb tourism. Concepts and implications for strategic planning. Annals of Tourism Research, 10, 2, pp. 239-263. https://doi. org/10.1016/0160-7383(83)90028-2

Grifols, M. A. (2003). Ulixes 21. Por un turismo sostenible en el Mediterráneo. $A$ Distancia, 21, 1, pp. 93-100.

Macpherson Mayol, E. (2000). Recursos Naturales. Arbor, 164, 653, pp. 37-49. https://doi.org/10.3989/arbor.2000. i653.1000

Noguera Tur, J., Ferrándis Martínez, A. y Riera Spiegelhalder, M. (2012). De complemento a motor: la transformación de la función del ocio y turismo en las estrategias de desarrollo local. El caso de la recuperación y valorización del patrimonio cultural. Arbor, 188, 754, 
pp. 379-393. https://doi.org/10.3989/ arbor.2012.754n2010

Pulido Fernández, J. I. (2003). Turismo de naturaleza y sostenibilidad. A Distancia, 21, 1, pp. 32-46.

Rivas García, J. y Magadán Díaz, M. (2008). Planificación y Gestión Sostenible del Turismo, Oviedo: Septem ediciones.

Sancho, A. y Buhalis, D. (dirs.) (1998). Introducción al turismo. Madrid: Organización Mundial del Turismo. Disponible en: https://asesoresenturismoperu.files. wordpress.com/2016/03/81-introduccionalturismo_omt.pdf

Valdés Peláez, L. (1996). El turismo rural en España. En: Pedreño Muñoz, A., Aguiló Pérez, E. y Monfort Mir, V. (eds.). Introducción a la economía del turismo en España. Madrid: Civitas.

Zárate Martín, M. A. (2012). Paisaje, forma y turismo en ciudades históricas. Estudios Geográficos, 73, 273, pp. 657694. https://doi.org/10.3989/estgeogr.201223

\section{Otros recursos}

Carta del Turismo Sostenible. Conferencia Mundial de Turismo Sostenible. Lanzarote, Islas Canarias, España, 27 y 28 de abril de 1995. Disponible en: http:// www.e-unwto.org/doi/abs/10.18111/ unwtodeclarations.1995.21.15.1

Carta Europea de Turismo Sostenible en los Espacios Protegidos. Federación EUROPARC. Disponible en: http://www. european-charter.org
Carta Internacional sobre Turismo Cultural. La Gestión del Turismo en los sitios con Patrimonio Significativo. Internacional Council on Monuments and Sites (ICOMOS). Disponible en: http://www.international.icomos.org/charters/tourism_sp.pdf

Carta Mundial de Turismo Sostenible +20 . Disponible en: http://cartamundialdeturismosostenible2015.com/wp-content/uploads/2016/05/Carta-Mundialde-Turismo-Sostenible-20.pdf

Ecoturismo. Disponibleen:https://es.wikipedia. org/wiki/Turismo_ecol\%C3\%B3gico

Observación de aves. Disponible en https:// es.wikipedia.org/wiki/Observación_ de_aves

Organización Mundial del Turismo (UNWTO). Código Ético Mundial para el Turismo. Por un Turismo responsable. Naciones Unidas, 2001. Disponible en: http://cf.cdn.unwto.org/sites/all/files/ docpdf/gcetbrochureglobalcodees.pdf

Organización Mundial del Turismo (UNWTO). Sustainable Development of Tourism. Definición. Disponible en: http:// sdt.unwto.org/es/content/definicion

Organización Mundial del Turismo (UNWTO). Yachana Lodge. Disponible en: http:// www2.unwto.org/es/node/36262

Organización Mundial del Turismo (UNWTO). Panorama OMT del turismo internacional. Edición 2016. Disponible en: http://www.e-unwto.org/doi/ pdf/10.18111/9789284418152
Plan Nacional de Paisaje Cultural. Instituto del Patrimonio Cultural de España, 2012. Disponible en: http://www.mecd. gob.es/planes-nacionales/planes-nacionales/paisaje-cultural.html

Real Decreto 416/2014, de 6 de junio, por el que se aprueba el Plan sectorial de turismo de naturaleza y biodiversidad 2014-2020. Boletín Oficial del Estado núm. 147, de 18 de junio de 2014, pp. 46026-46048. Disponible en: https://www.boe.es/diario_boe/txt. php?id=BOE-A-2014-6432

Tejera, J. Africa Scientia 3/4: Turismo responsable en Namibia y Lesoto. Ecotumismo. Disponible en: http://www.ecotumismo.org/turismo-responsable/turismo/ africa-scientia-34-turismo-responsableafrica-en-namibia-y-lesoto/

The International Ecotourism Society (TIES). Ecoturismo. Disponible en: http://www. ecotourism.org/

The International Ecotourism Society (TIES). Hoja Informativa: Ecoturismo Global. Disponible en http://www.southernnature.cl/sala_lectura/turismo/ TIES_Ecoturismo_Global.pdf

The Namibian Community Based Tourism Association - Asociación Namibia de Turismo con Base Comunitaria. Foro para el turismo sostenible. Disponible en: http://www.nacobta.com.na/ 